\title{
Impact of the COVID-19 pandemic on radiology department emergency ultrasound utilization
}

\author{
Mark D. Messina ${ }^{1}$ (D) Marjorie W. Stein ${ }^{1} \cdot \operatorname{lan}$ J. Armstrong ${ }^{1} \cdot$ Ellen L. Wolf $^{1}$
}

Received: 16 February 2021 / Accepted: 21 April 2021 / Published online: 29 April 2021

(c) American Society of Emergency Radiology 2021

\begin{abstract}
Purpose To analyze the change in utilization of healthcare resources through a review of ultrasound examinations performed in the emergency department of an urban healthcare system in NYC during the time of peak COVID-19 outbreak.

Methods This is a retrospective review analyzing ED ultrasound exams performed by the radiology department of an urban healthcare system during the 8-week time period of the peak COVID-19 outbreak in NYC, compared to a time-matched period one year prior. Data regarding the examination type and indication were obtained in addition to patient demographics and indicators of outcomes including admission, length of stay, and mortality.

Results There was a 58\% decrease in ED ultrasounds performed by the radiology department during the COVID-19 time period. Exams performed during the pandemic compared to the pre-pandemic period were more likely to be performed on men ( 28.3 vs $18.0 \%, p<0.01$ ), older patients ( 36 vs. 35 years, $p=0.02)$, and patients subsequently admitted ( 17.8 vs. $13.4 \%$, $p=0.03)$. There was also a difference in the distribution of exam type $(p=0.01)$. There was no difference in death, rate of surgery/intervention performed, or distribution of clinical indication. When correcting for gender, there was only an increase in studies leading to hospital admission in the female-only group (14.9 vs. $10.7 \%, p=0.05$ ).

Conclusion COVID-19 had a drastic impact on the utilization of emergency department ultrasounds performed by the radiology department, with a decrease in total exams performed and changes in patient demographics, including a higher proportion of male patients and increases in some markers of disease severity, including rate of hospital admission.
\end{abstract}

Keywords Emergency radiology $\cdot$ Ultrasound $\cdot$ COVID-19 · Imaging utilization

\section{Introduction}

COVID-19, the disease caused by severe acute respiratory syndrome coronavirus 2 (SARS-CoV-2), was declared a pandemic by the World Health Organization on March 11, 2020 [1]. Within one month of that declaration, 110,000 cases of COVID-19 were reported in New York City (NYC) [2]. Emergency departments (ED) and hospitals became inundated with patients with COVID-like symptoms while simultaneously, ED visits for other presentations dropped dramatically. Efforts by local governmental officials to minimize hospital bed utilization for non-COVID purposes were accomplished by temporary stay orders on non-critical surgeries and medical procedures, as

Mark D. Messina

MaMessina@montefiore.org

1 Department of Radiology, Montefiore Medical Center, Albert Einstein College of Medicine, 111 E 210th Street, Bronx, NY 10467, USA well as loosening regulation on remote interaction with healthcare providers, such as the use of telemedicine. Patient attitudes towards pursuing medical care were undoubtedly influenced by fear of contraction of COVID-19 as the pandemic threatened to overwhelm city hospitals. This project aims to analyze the change in utilization of healthcare resources through a review of ultrasound examinations performed in the ED setting by the radiology department of an urban medical healthcare system in NYC during the time of peak COVID outbreak compared to one year prior to the pandemic with the goal of analyzing differences in patient characteristics and outcomes, exams performed, and clinical indications.

\section{Materials and methods}

This study was performed within Montefiore Health System, an academic health care system centered in the Bronx, New York. We retrospectively identified all 
ultrasound examinations performed by the radiology department on ED patients during an 8-week time period starting on March 15, 2020, corresponding to the rise and peak time period of the COVID-19 pandemic in NYC. We chose a sampling of cases during the identical time frame in 2019 as a control group using every fourth case completed during the time frame in order to equalize the number of exams across both study populations. We included the examinations performed in the EDs of three of our hospitals in the Bronx and included only adult patients, aged 21 and older. In patients who had multiple ultrasound examinations during one ED visit, each study was counted individually. Three patients were represented in both the 2019 and 2020 groups and were removed from analysis. We obtained information from the electronic medical record regarding patient age and gender, as well as type of examination performed and indication. Information regarding patient outcomes including hospital admission, length of hospital stay, mortality, and procedural interventions performed was collected. COVID testing and positivity status were obtained for the 2020 group. Emergency department patient volume and number of patients admitted were obtained for the time frames of analysis. In order to facilitate analysis, multiple study types and indications for examinations were consolidated as outlined in Tables 1 and 2. For statistical analysis, we used Wilcoxon ranked sum tests for continuous variables and chi-squared tests for categorical variables to determine differences between the two years. This study was approved by the institutional review board.

\section{Results}

Study results are outlined in Tables 3, 4, 5, and 6. In the 8 -week period starting on March 15, 2020, there were 639 ultrasound examinations performed on adult ED patients by the radiology department fitting the study criteria. There were 1511 ultrasound examinations performed in the same 8-week period in 2019 , out of which 640 were selected for analysis by study protocol. $181(28.3 \%)$ of the studies in 2020 were performed on males compared to $115(18.0 \%)$ in the 2019 group $(p<0.01)$. The median age of patients on which studies were performed in 2020 was 36 compared to 35 in $2019(p=0.02)$. Of the studies performed in 2020, $114(17.8 \%)$ were followed by hospital admission, compared to $86(13.4 \%)$ in $2019(p=0.03)$. There were 3 deaths in the 2020 group and 0 deaths in the 2019 group $(p=0.83)$. Forty-five $(7.0 \%)$ of the studies performed in 2020 preceded a procedural intervention during that ED visit or subsequent hospital stay, as opposed to 47 $(7.3 \%)$ in the 2019 group $(p=0.84)$. The average length of hospitalization for both groups was 3 days $(p=0.38)$. The distribution of study type was significantly different between the two years $(p=0.01)$. In both 2020 and 2019, obstetric ultrasound was the most commonly performed exam type (27.1 and $30.2 \%$ respectively). In 2020 and 2019 respectively, abdominal ultrasound represented 25.2 and $22.2 \%$ of studies, female non-obstetric pelvic ultrasound represented 16.4 and $23.6 \%$, scrotum and penile ultrasound represented 8.3 and $5.0 \%$, and extremity duplex studies represented 8.9 and $5.5 \%$. There was no significant difference in the clinical indications for examinations between the years $(p=0.06)$. In 2020 and 2019 respectively, abdominal pain represented 31.3 and $29.1 \%$, vaginal symptoms represented 18.6 and $18.9 \%$, and pelvic pain represented 13.5 and $19.4 \%$. When completing the analysis correcting for gender, there was no significant difference for the male-only group in age, admission rate, death, procedural intervention, length of stay, distribution of study type, or composition mix of clinical indication. There was a significant increase in studies leading to hospital admission in the female-only group, with 68 studies $(14.9 \%)$ leading to admission in 2020 compared to 56 (10.7\%)

Table 1 Exam type category. Original ultrasound examination types and respective consolidated categories used for analysis

\begin{tabular}{lc}
\hline Original exam types & Consolidated category \\
\hline $\begin{array}{l}\text { Abdomen complete + RUQ + LUQ + Miscellaneous abdomen (Aorta/IVC duplex + Ascites evaluation + Gallblad- } \\
\text { der + Hepatic duplex + Renal artery duplex + Pancreas) }\end{array}$ & Abdomen \\
$\begin{array}{l}\text { Lower extremity duplex bilateral + Lower extremity duplex unilateral + Upper extremity duplex bilateral + Upper } \\
\text { extremity duplex unilateral }\end{array}$ & Extremity duplex \\
Pelvis TA/TV + Pelvis TA + Pelvis TV & Non-OB female pelvis \\
Obstetric TA/TV + Obstetric TA + Obstetric TV & OB \\
Renal and bladder + Renal unilateral + Bladder & Renal and bladder \\
Right lower quadrant & Right lower quadrant \\
Scrotum + Penis & Scrotum and penis \\
Extremity soft tissue + Chest wall + Abdominal wall + Neck + Thyroid + Breast + Axilla & Soft Tissue \\
Pancreas transplant + Renal transplant & Transplant \\
\hline
\end{tabular}


Table 2 Indication for exam. Original clinical indications for ultrasound examinations and respective consolidated categories used for analysis

\begin{tabular}{ll}
\hline Original indications & Consolidated category \\
\hline Abdominal pain + Epigastric pain + LLQ pain + RUQ pain & Abdominal pain \\
Abnormal same day imaging + Follow up abnormal outside imaging & Abnormal imaging \\
Anemia + Elevated LFTs + Elevated lipase + Thrombocytopenia & Abnormal labs \\
Breast mass + Breast pain & Breast symptoms \\
Leg pain + Leg swelling + Upper extremity pain + Upper extremity swelling & Extremity vascular symptoms \\
Abscess + Hyperemesis + Hypertension + Neck swelling + Trauma & Miscellaneous \\
Pelvic pain & Pelvic pain \\
Follow up OB US + Pregnancy evaluation without OB complaint & Pregnancy evaluation \\
RLQ pain & RLQ pain \\
Penis pain + Scrotal pain + Scrotal swelling & Scrotum/penis symptoms \\
AKI + Back pain + Dysuria + Flank pain + Hematuria + Urinary retention & Urinary symptoms \\
Vaginal bleeding + Vaginal discharge + Vaginal pain & Vaginal symptoms \\
\hline
\end{tabular}

in $2019(p=0.05)$. Of the studies performed in the 2020 group, there were 142 COVID PCR tests performed in the ED and 36 of those tests $(25.4 \%)$ were positive. Twentyfive patients had a positive COVID test prior to their ED visit. There was a $41.3 \%$ decrease in emergency department volume from the 2019 study time period compared to the 2020 time period and a $35.1 \%$ decrease in the number of patients admitted to the hospital directly from the ED. The percentage of all ED patients admitted to the hospital during the study time period in 2019 was $38.6 \%$, compared to $42.7 \%$ in the same time period in $2020(p<0.01)$.

\section{Discussion}

COVID-19 caused a dramatic change in healthcare utilization throughout the United States and indeed throughout the world. In the month of April 2020 alone, there were 16,395

Table 3 Study characteristics. Study characteristics for emergency ultrasound examinations performed by the radiology department in the 8-week time period of peak COVID-19 pandemic in NYC (March 11, 2020 to May 2, 2020) compared to a sampling of exams performed in the same 8-week time period one year prior to the pandemic

\begin{tabular}{lllr}
\hline & $2019(n=640)$ & $2020(n=639)$ & $p$-value \\
\hline Admission & $86(13.4 \%)$ & $114(17.8 \%)$ & 0.0302 \\
Procedural intervention & $47(7.3 \%)$ & $45(7.0 \%)$ & 0.8347 \\
Death & $0(0 \%)$ & $3(0.5 \%)$ & 0.0827 \\
Age $^{\mathrm{a}}$ & $35(27-47)$ & $36(28-52)$ & 0.0167 \\
Male $_{\text {Length of }}$ & $115(18.0 \%)$ & $181(28.3 \%)$ & $<0.0001$ \\
hospitalization $^{\mathrm{b}}$ & $3(2-5)$ & $3(1-6)$ & 0.3799 \\
\hline
\end{tabular}

${ }^{\text {a }}$ Value given as median years (inter-quartile range)

b Value given as median days (inter-quartile range) deaths in NYC attributed to COVID-19, more than any single state death toll from COVID in the first six months since it was declared a pandemic [2]. The Bronx, where our healthcare system is located, was particularly hard hit in the first wave of the pandemic. The Bronx is often regarded as the most socioeconomically disadvantaged borough of NYC. As compared to the other four boroughs, the Bronx has the lowest median household income, lowest percentage of high school and college graduates [3], and highest percentage of premature deaths (before the age of 75) [4]. As of the end of April 2020, the Bronx had the highest percentage of inhabitants testing positive for COVID-19 antibodies at $27.6 \%$, compared to $19.9 \%$ for NYC as a whole, and $12.3 \%$ for the state of New York [5].

Regulatory measures were taken by federal, state, and local agencies in an attempt to slow the spread of the virus, primarily focused on the idea of a modified "lockdown" including closure of non-essential businesses and schools [6]. The practice of medicine was drastically changed as

Table 4 Ultrasound test type. Type of ultrasound examinations performed on emergency department patients by the radiology department in the 8-week time period of peak COVID-19 pandemic in NYC compared to a sampling of exams performed in the same 8-week time period one year prior to the pandemic

\begin{tabular}{llll}
\hline & $2019(n=640)$ & $2020(n=639)$ & $p=0.0060$ \\
\hline Abdomen & $142(22.2 \%)$ & $161(25.2 \%)$ & \\
Extremity duplex & $35(5.5 \%)$ & $57(8.9 \%)$ & \\
OB & $193(30.2 \%)$ & $173(27.1 \%)$ \\
Non-OB female pelvis & $151(23.6 \%)$ & $105(16.4 \%)$ \\
RLQ & $8(1.3 \%)$ & $6(0.9 \%)$ \\
Renal and/or bladder & $63(9.8 \%)$ & $68(10.6 \%)$ \\
Soft tissue & $12(1.9 \%)$ & $12(1.9 \%)$ \\
Scrotum/penis & $32(5.0 \%)$ & $53(8.3 \%)$ \\
Transplant & $4(0.6 \%)$ & $4(0.6 \%)$ & \\
\hline
\end{tabular}


Table 5 Indication for exam. Clinical indications for emergency ultrasound examinations performed by the radiology department in the 8 -week time period of peak COVID-19 pandemic in NYC compared to a sampling of exams performed in the same 8-week time period one year prior to the pandemic

\begin{tabular}{|c|c|c|c|}
\hline & $2019(N=640)$ & $2020(N=639)$ & $p=0.0597$ \\
\hline Abdominal pain & $186(29.1 \%)$ & $200(31.3 \%)$ & \\
\hline Abnormal imaging & $29(4.5 \%)$ & $21(3.3 \%)$ & \\
\hline Abnormal labs & $11(1.7 \%)$ & $13(2.0 \%)$ & \\
\hline Breast symptoms & $5(0.8 \%)$ & $4(0.6 \%)$ & \\
\hline $\begin{array}{l}\text { Extremity vascular } \\
\text { symptoms }\end{array}$ & $37(5.8 \%)$ & $54(8.5 \%)$ & \\
\hline Miscellaneous & $10(1.6 \%)$ & $8(1.3 \%)$ & \\
\hline Pelvic pain & $124(19.4 \%)$ & $86(13.5 \%)$ & \\
\hline Pregnancy evaluation & $10(1.6 \%)$ & $9(1.4 \%)$ & \\
\hline RLQ pain & $15(2.3 \%)$ & $8(1.3 \%)$ & \\
\hline $\begin{array}{l}\text { Scrotum/penis symp- } \\
\text { toms }\end{array}$ & $32(5.0 \%)$ & $51(8.0 \%)$ & \\
\hline Urinary symptoms & $60(9.4 \%)$ & $66(10.3 \%)$ & \\
\hline Vaginal symptoms & $121(18.9 \%)$ & $119(18.6 \%)$ & \\
\hline
\end{tabular}

well. Elective surgeries were indefinitely postponed, as were most outpatient office visits and medical procedures, including screening and otherwise routine imaging tests $[7,8]$.

While hospital resources were stretched thin by the acuity of COVID-19, a decrease in ED utilization is now well documented, especially regarding non-COVID related illnesses [9-12]. In the hospitals used in this analysis, there was a total of 32,899 emergency department visits in the selected 2019 time period compared to 19,318 in the 2020 time period, a decrease of $41.3 \%$. A decline in the use of diagnostic imaging in the ED setting during the pandemic, particularly cross-sectional imaging, has also been documented. Studies have shown a significant decrease in the utilization of ED head CT examinations [13], abdominopelvic CT examinations [14], and overall ED imaging [15] in North American institutions during the COVID pandemic.

There were concerns among healthcare workers that members of the public who needed medical care were avoiding hospitals, presumably at least in part out of fear of infection. The NYC Department of Health and Mental Hygiene reported 24,172 deaths in excess of the seasonal expected baseline between March 11 and May 2 of 2020, with 22\% of those deaths not identified as being COVID-19 associated [16]. Reports of decreased rates of diagnoses of specific nonCOVID illnesses with increased disease severity, such as acute appendicitis and strokes, have been described [17-19]. While the emergency department volume of our hospitals decreased in the pandemic period, the percentage of ED visits resulting in hospital admission rose, likely due to the higher acuity of COVID patients. In the pre-pandemic study period, $38.6 \%$ of ED visits resulted in admission, compared to $42.7 \%$ in the 2020 period.
In our 8-week time frame in 2020 representing the rise and peak of the COVID-19 pandemic in NYC, there were 639 ultrasound exams performed on ED patients by the radiology department fitting study criteria, a 57.7\% decrease from the 1511 exams performed in the same 8-week time period one year prior. In comparing 2020 to 2019 , there were statistically significant increases in the percentage of ultrasound exams performed on males and in patients subsequently admitted to the hospital. A statistically significant increase in patient age from 2019 to 2020 was shown, although this is unlikely to have clinical significance as the difference between the two cohorts was only one year of age (36 in 2020 vs. 35 in 2019). There was also a statistically significant difference in the distribution of type of studies performed between the two years. However, there was no significant difference in number of studies that preceded procedural interventions, length of hospital stay of admitted patients, and distribution of clinical indications for exams. After correcting for gender, only an increase in studies leading to hospital admission demonstrated statistical significance for females.

An association between male gender and COVID-19 infection, severity of disease, and hospitalization rate has been highlighted since early in the course of the pandemic [20-22]. A positive association between male gender and imaging utilization during the COVID pandemic has also been described [23]. In our study, we see a higher proportion of ultrasounds performed in men during the COVID time period compared to the pre-pandemic period. Our study demonstrated a statistically significant difference in the admission rate in patients receiving diagnostic ultrasound exams between 2019 and 2020, which can at least in part be attributed to the fact that many patients in 2020 were reluctant to go to an emergency room due to fear of COVID exposure and those who did go were indeed sicker and more likely to be admitted. Interestingly, when analyzing males independently, there was no statistically significant difference in the admission rate of males between the two years, which is more difficult to explain. Additionally, the rate of admission of patients receiving ultrasound exams is noted to be lower than the admission rate for all ED patients during both the selected 2019 and 2020 time periods; this is likely due to the utilization of ultrasound for typically low admission-risk conditions such as pregnancy, pelvic pain, vaginal bleeding, and flank pain. As previously mentioned, the all-comer emergency department admission rate was $38.6 \%$ in the pre-pandemic period and $42.7 \%$ in pandemic time period, compared to 13.4 and $17.8 \%$, respectively, for patients undergoing ultrasound exams by the radiology department.

While the composition mix of studies when analyzing males alone did not demonstrate a significant difference in our population, there was a decrease in the percentage of abdomen exams performed on males in the 2020 population (36.5\%) compared to 2019 (40.9\%) with an increase in extremity duplex exams (10.5 vs. $6.1 \%$ ). The female-only group also 
Table 6 Patient and study characteristics by gender. Patient and study characteristics for ultrasound examinations performed in the emergency department by the radiology department during the peak-
COVID-19 pandemic in NYC compared a time-matched sampling one year prior to the pandemic, analyzed by gender

\begin{tabular}{|c|c|c|c|c|c|c|}
\hline & \multicolumn{3}{|l|}{ Male } & \multicolumn{3}{|l|}{ Female } \\
\hline & $2019(n=115)$ & $2020(n=181)$ & $p$-value & $2019(n=525)$ & $2020(n=458)$ & $p$-value \\
\hline Admission & $30(26.1 \%)$ & $46(25.4 \%)$ & 0.8973 & $56(10.7 \%)$ & $68(14.9 \%)$ & 0.0489 \\
\hline Surgery/intervention & $13(11.3 \%)$ & $18(9.9 \%)$ & 0.7096 & $34(6.5 \%)$ & $27(5.9 \%)$ & 0.7064 \\
\hline Death & $0(0 \%)$ & $2(1.1 \%)$ & 0.2580 & $0(0 \%)$ & $1(0.2 \%)$ & 0.2841 \\
\hline $\operatorname{Age}^{\mathrm{a}}$ & $47(32-63)$ & $51(35-61)$ & 0.8176 & $33(26-42)$ & $33(27-46)$ & 0.2796 \\
\hline Length of hospital stay ${ }^{b}$ & $3(2-6)$ & $4(2-9)$ & 0.4636 & $2(1-3.5)$ & $2.5(1-5)$ & 0.7887 \\
\hline Ultrasound test type: & & & 0.7257 & & & 0.3126 \\
\hline Abdomen & $47(40.9 \%)$ & $66(36.5 \%)$ & & $95(18.1 \%)$ & $95(20.7 \%)$ & \\
\hline Extremity duplex & $7(6.1 \%)$ & $19(10.5 \%)$ & & $28(5.3 \%)$ & $38(8.3 \%)$ & \\
\hline $\mathrm{OB}$ & $0(0 \%)$ & $0(0 \%)$ & & $192(36.8 \%)$ & $173(37.8 \%)$ & \\
\hline Non- OB female pelvis & $0(0 \%)$ & $0(0 \%)$ & & $151(28.8 \%)$ & $105(22.9 \%)$ & \\
\hline RLQ & $0(0 \%)$ & $2(1.1 \%)$ & & $8(1.5 \%)$ & $4(0.9 \%)$ & \\
\hline Renal and/or bladder & $24(20.9 \%)$ & $35(19.3 \%)$ & & $39(7.4 \%)$ & $33(7.2 \%)$ & \\
\hline Soft tissues & $2(1.7 \%)$ & $3(1.7 \%)$ & & $10(1.9 \%)$ & $9(2.0 \%)$ & \\
\hline Scrotum/penis & $32(27.8 \%)$ & $53(29.3 \%)$ & & $0(0 \%)$ & $0(0 \%)$ & \\
\hline Transplant & $3(2.6 \%)$ & $3(1.7 \%)$ & & $1(0.2 \%)$ & $1(0.2 \%)$ & \\
\hline Indication for exam: & & & 0.8214 & & & 0.4963 \\
\hline Abdominal pain & $36(31.3 \%)$ & $59(32.6 \%)$ & & $150(28.6 \%)$ & $141(30.8 \%)$ & \\
\hline Abnormal imaging & $5(4.4 \%)$ & $6(3.3 \%)$ & & $24(4.6 \%)$ & $15(3.3 \%)$ & \\
\hline Abnormal labs & $4(3.5 \%)$ & $10(5.5 \%)$ & & $7(1.3 \%)$ & $3(0.7 \%)$ & \\
\hline Breast symptoms & $0(0 \%)$ & $0(0 \%)$ & & $5(1.0 \%)$ & $4(0.9 \%)$ & \\
\hline Extremity symptoms & $7(6.1 \%)$ & $17(9.4 \%)$ & & $30(5.7 \%)$ & $37(8.1 \%)$ & \\
\hline Miscellaneous & $3(2.6 \%)$ & $3(1.7 \%)$ & & $7(1.3 \%)$ & $5(1.1 \%)$ & \\
\hline Pelvic pain & $2(1.7 \%)$ & $2(1.1 \%)$ & & $122(23.2 \%)$ & $84(18.3 \%)$ & \\
\hline Pregnancy evaluation & $0(0 \%)$ & $0(0 \%)$ & & $10(1.9 \%)$ & $9(2.0 \%)$ & \\
\hline RLQ pain & $1(0.9 \%)$ & $0(0 \%)$ & & $14(2.7 \%)$ & $8(1.8 \%)$ & \\
\hline Scrotum/penis symptoms & $32(27.8 \%)$ & $51(28.2 \%)$ & & $0(0 \%)$ & $0(0 \%)$ & \\
\hline Urinary symptoms & $25(21.7 \%)$ & $33(18.3 \%)$ & & $35(6.7 \%)$ & $33(7.2 \%)$ & \\
\hline Vaginal symptoms & $0(0 \%)$ & $0(0 \%)$ & & $121(23.1 \%)$ & $119(26.0 \%)$ & \\
\hline
\end{tabular}

${ }^{\text {a } V a l u e ~ g i v e n ~ a s ~ m e d i a n ~ y e a r s ~(i n t e r-q u a r t i l e ~ r a n g e) ~}$

${ }^{\mathrm{b}}$ Value given as median days (inter-quartile range)

demonstrated a relative increase in extremity duplex exams, $5.3 \%$ of the study population in 2019 compared to $8.3 \%$ in 2020. Thromboembolism is an established complication of COVID-19 [24, 25] so an increase in the percentage of these examinations performed would be an expected finding. In the pandemic cohort, there were 57 extremity duplex exams performed and 8 of these studies demonstrated venous thromboses. In the pre-pandemic period, 35 extremity duplex studies were included in the analysis and one was positive for venous thrombosis. The study population does not include all patients receiving extremity duplex examinations in the emergency setting, as vascular surgery manages extremity duplex examinations in two of the three hospitals used in the analysis.

It is possible that the change in gender mix of our patient population is at least in part due to a relative decrease in exams performed on females in 2020 compared to 2019.
While the composition mix of both study type and clinical indication for females was not statistically different between the years, the percentage of non-obstetric pelvic ultrasounds decreased from $28.8 \%$ in 2019 to $22.9 \%$ in 2020 and the percentage of cases with pelvic pain as the indication decreased from $23.2 \%$ in 2019 to $18.3 \%$ in 2020 , suggesting that nongravid women presenting with pelvic pain may in part be responsible for the difference in gender mix between the two years. This could be due to fewer non-pregnant women presenting to the ED with pelvic pain or ED providers choosing to defer these examinations in a time of pandemic.

It has been suggested that the COVID-19 pandemic may lead to a decrease in the fertility rate $[26,27]$. While the number of ultrasound exams in our study completed in 2020 was nearly $60 \%$ decreased compared to the same time frame in 2019, the percentage of obstetric studies performed out of all women was 
similar (37.8\% in 2020 vs. $36.8 \%$ in 2019). Given that radiology department-directed ED ultrasounds are predominantly from the first trimester and that this study time frame was early in the course of the pandemic in the USA, it is not surprising that any potential change in fertility rate was not yet seen.

It is important to reference the change in emergency department volume when discussing changes in imaging utilization. The ED volume of the hospitals analyzed decreased $41.3 \%$ from the 2019 time period compared to the 2020 period. This decrease is less than the change in volume of ED ultrasounds performed by the radiology department, which neared a $60 \%$ decline. The relative decrease in utilization of radiology department ultrasound during the pandemic was certainly accentuated by the change in distribution of clinical presentations to the ED [28] and in practitioner decisions to defer imaging of lower acuity patients [14, 29]. Another factor leading to our decreased ED imaging utilization in 2020 can be understood by the study population we serve. Because our healthcare system is located in an underserved, urban neighborhood, our ED is not only used as an acute care facility, but also as a primary care facility as many of our patients do not have primary care providers. It is common practice at our institution to perform some diagnostic exams that, although not emergent, have an otherwise low likelihood of being completed in the outpatient setting at a later date. At the height of the pandemic, this practice was presumably altered.

This study has several limitations. The study was performed retrospectively and is limited to three of our health system's inner-city hospital emergency departments. In our health system, not all ultrasound examinations are performed by the radiology department; for example, emergency department point of care ultrasound is performed in selected patients and is not captured in this analysis. Our health system experienced a high volume of patients with COVID-19 infections during the timeframe used for analysis which may limit the generalizability of our results. Using a timeframe of the first 8 weeks of the pandemic may also limit generalizability, as emergency departments across the world had to learn to quickly adapt to operating within a pandemic, which altered workflow and decision-making processes.

\section{Conclusion}

COVID-19 has caused a dramatic change in healthcare utilization. Our study shows a 58\% decrease in the volume of ultrasounds performed by the radiology department on ED patients during the rise and peak of the pandemic at a severely impacted health system in NYC. This decrease outpaced the decline in ED volume during our time periods of analysis. In our population, there was a significant increase in percentage of exams performed on males during the pandemic. Additionally, there was a mixed impact in markers of disease severity with an increase in proportion of patients subsequently admitted and no significant change in mortality, rate of procedural intervention, or length of hospitalization. A difference in the types of ultrasound exams performed between the groups was seen, with a relative increase in extremity duplex examinations and abdominal ultrasounds during the pandemic period coupled with a relative decrease in non-obstetric pelvic ultrasounds. The cause of the differences found in this study is likely multifactorial and includes governmental efforts to reduce hospital utilization, healthcare providers' decisions on the appropriateness of diagnostic examinations in a period of pandemic, and viral fear.

Author contribution All authors contributed to the study conception/ design, data collection, and analysis. The first draft of the manuscript was written by Mark Messina, MD and all authors commented on previous versions of the manuscript. All authors read and approved the final manuscript.

\section{Declarations}

Ethics approval and consent to participate This study was reviewed and approved by the Montefiore Medical Center/Albert Einstein College of Medicine Institutional Review Board. An IRB waiver was obtained.

Consent for publication This study was reviewed and approved by the Montefiore Medical Center/Albert Einstein College of Medicine Institutional Review Board. An IRB waiver was obtained.

Conflict of interest The authors declare that they have no conflict of interest.

Code availability Not applicable.

\section{References}

1. World Health Organization (2020) WHO Director-General's opening remarks at the media briefing on COVID-19. World Health Organization Director General. https://www.who.int/ director-general/speeches/detail/who-director-general-s-openi ng-remarks-at-the-media-briefing-on-covid-19-11-march2020. Accessed 09/01/2020

2. Center for Disease Control Case Surveillance Task Force (2020) COVID-19 Case Surveillance Public Use Data. https://data.cdc. gov/Case-Surveillance/COVID-19-Case-Surveillance-PublicUse-Data/vbim-akqf. Accessed 09/05/2020

3. United States Census Bureau (2019) Quick Facts, New York City, New York. United States Department of Commerce. https://www.census.gov/quickfacts/fact/table/newyorkcitynewy ork, bronxcountybronxboroughnewyork,kingscountybrooklynb oroughnewyork, newyorkcountymanhatt anboroughnewyor $\mathrm{k}$,queenscountyqueensboroughnewyork,richmondcountystaten islandboroughnewyork/PST045219. Accessed 09/05/2020

4. New York State Department of Health (2017) County Health Indicators by Race/Ethnicity (CHIRE). https://www.health.ny. 
gov/statistics/community/minority/county/index.htm. Accessed 09/05/2020

5. Office of the Governor of New York (2020) Amid Ongoing COVID-19 Pandemic, Governor Cuomo Announces Results of Completed Antibody Testing Study of 15,000 People Showing 12.3 Percent of Population Has COVID-19 Antibodies. New York State. https://www.governor.ny.gov/news/amid-ongoingcovid-19-pandemic-governor-cuomo-announces-results-compl eted-antibody-testing. Accessed 09/12/2020

6. Office of the Governor of New York (2020) Governor Cuomo Signs the 'New York State on PAUSE' Executive Order. New York State. https://www.governor.ny.gov/news/governorcuomo-signs-new-york-state-pause-executive-order. Accessed $09 / 12 / 2020$

7. Office of the Governor of New York (2020) Continuing Temporary Suspension and Modification of Laws Relating to the Disaster Emergency. New York State. https://www.governor.ny.gov/ news/no-20225-continuing-temporary-suspension-and-modif ication-laws-relating-disaster-emergency Accessed 09/12/2020

8. Torous J, Jän Myrick K, Rauseo-Ricupero N, Firth J (2020) Digital mental health and COVID-19: using technology today to accelerate the curve on access and quality tomorrow. JMIR Ment Health 7(3):e18848. https://doi.org/10.2196/18848

9. Barten DG, Latten GHP, van Osch FHM (2020) Reduced emergency department utilization during the early phase of the COVID-19 pandemic: viral fear or lockdown effect? Disaster Med Public Health Prep:1-4. https://doi.org/10.1017/dmp.2020. 303

10. Hughes HEHT, Morbey R, Challen K, Oliver I, Smith GE, Elliot AJ (2020) Emergency department use during COVID19 as described by syndromic surveillance. Emerg Med J 37(10):600-604

11. Nunez JH, Sallent A, Lakhani K, Guerra-Farfan E, Vidal N, Ekhtiari S, Minguell J (2020) Impact of the COVID-19 pandemic on an emergency traumatology service: experience at a tertiary trauma centre in Spain. Injury 51(7):1414-1418. https:// doi.org/10.1016/j.injury.2020.05.016

12. Cohen BA, Wessling EG, Serina PT, Cruz DS, Kim HS, McCarthy DM, Loftus TM (2020) Emergency department operations in a large health system during COVID-19. Am J Emerg Med. https://doi.org/10.1016/j.ajem.2020.05.097

13. Agarwal M, Udare A, Alabousi A, van der Pol CB, Ramonas L, Mascola K, Edmonds B, Ramonas M (2020) Impact of the COVID-19 pandemic on emergency CT head utilization in Ontario-an observational study of tertiary academic hospitals. Emerg Radiol 27(6):791-797. https://doi.org/10.1007/ s10140-020-01857-3

14. Gibson AL, Chen BY, Rosen MP, Paez SN, Lo HS (2020) Impact of the COVID-19 pandemic on emergency department CT for suspected diverticulitis. Emerg Radiol 27(6):773-780. https://doi.org/10.1007/s10140-020-01865-3

15. Parikh KD, Ramaiya NH, Kikano EG, Tirumani SH, Pierce J, Butcher C, Sunshine JL, Plecha DM (2020) Quantifying the decrease in emergency department imaging utilization during the COVID-19 pandemic at a multicenter healthcare system in Ohio. Emerg Radiol 27(6):765-772. https://doi.org/10.1007/ s10140-020-01848-4

16. New York City Department of Health and Mental Hygiene (DOHMH) COVID-19 Response Team (2020) Preliminary estimate of excess mortality during the COVID-19 outbreak - New York City, March 11-May 2, 2020. MMWR Morb Mortal Wkly Rep 69(19):603-605. https://doi.org/10.15585/mmwr.mm691 $9 \mathrm{e} 5$

17. Romero J, Valencia S, Guerrero A (2020) Acute appendicitis during coronavirus disease 2019 (COVID-19): changes in clinical presentation and CT findings. J Am Coll Radiol 17(8):1011-1013. https://doi.org/10.1016/j.jacr.2020.06.002
18. O’Brien CM, Jung K, Dang W, Jang HJ, Kielar AZ (2020) Collateral damage: the impact of the COVID-19 pandemic on acute abdominal emergency presentations. J Am Coll Radiol 17(11):1443-1449. https://doi.org/10.1016/j.jacr.2020.08.010

19. Dula AN, Gealogo Brown G, Aggarwal A, Clark KL (2020) Decrease in stroke diagnoses during the COVID-19 pandemic: where did all our stroke patients go? JMIR Aging 3(2):e21608. https://doi.org/10.2196/21608

20. Kalyanaraman Marcello R, Dolle J, Grami S, Adule R, Li Z, Tatem K, Anyaogu C, Apfelroth S, Ayinla R, Boma N, Brady T, Cosme-Thormann BF, Costarella R, Ford K, Gaither K, Jacobson J, Kanter M, Kessler S, Kristal RB, Lieber JJ, Mukherjee V, Rizzo V Jr, Rowell M, Stevens D, Sydney E, Wallach A, Chokshi DA, Davis N, New York City Health + Hospitals C-PHDT (2020) Characteristics and outcomes of COVID-19 patients in New York City's public hospital system. PLoS ONE 15(12):e0243027. https://doi.org/10.1371/journal.pone.0243027

21. Wiersinga WJ, Rhodes A, Cheng AC, Peacock SJ, Prescott HC (2020) Pathophysiology, Transmission, diagnosis, and treatment of coronavirus disease 2019 (COVID-19): a review. JAMA 324(8):782-793. https://doi.org/10.1001/jama.2020.12839

22. Garg S, Kim L, Whitaker M, O'Halloran A, Cummings C, Holstein R, Prill M, Chai SJ, Kirley PD, Alden NB, Kawasaki B, Yousey-Hindes K, Niccolai L, Anderson EJ, Openo KP, Weigel A, Monroe ML, Ryan P, Henderson J, Kim S, Como-Sabetti K, Lynfield R, Sosin D, Torres S, Muse A, Bennett NM, Billing L, Sutton M, West N, Schaffner W, Talbot HK, Aquino C, George A, Budd A, Brammer L, Langley G, Hall AJ, Fry A (2020) Hospitalization rates and characteristics of patients hospitalized with laboratory-confirmed coronavirus disease 2019 - COVID-NET, 14 States, March 1-30,2020. MMWR Morb Mortal Wkly Rep 69(15):458-464. https://doi.org/10.15585/mmwr.mm6915e3

23. Naidich JJ, Boltyenkov A, Wang JJ, Chusid J, Rula E, Hughes D, Sanelli PC (2020) Imaging utilization during the COVID-19 pandemic highlights socioeconomic health disparities. J Am Coll Radiol. https://doi.org/10.1016/j.jacr.2020.10.016

24. Cai C, Guo Y, You Y, Hu K, Cai F, Xie M, Yang L, Ling K, Ye D, Misra S, Wang W, Li Y (2020) Deep venous thrombosis in COVID-19 patients: a cohort analysis. Clin Appl Thromb Hemost 26:1076029620982669. https://doi.org/10.1177/1076029620982669

25. Di Minno A, Ambrosino P, Calcaterra I, Di Minno MND (2020) COVID-19 and venous thromboembolism: a meta-analysis of literature studies. Semin Thromb Hemost 46(7):763-771. https:// doi.org/10.1055/s-0040-1715456

26. Luppi F, Arpino B, Rosina A (2020) The impact of COVID-19 on fertility plans in Italy, Germany, France, Spain, and the United Kingdom. Demogr Res 43(47):1399-1412

27. Lindberg LD, Bell DL, Kantor LM (2020) The sexual and reproductive health of adolescents and young adults during the COVID19 pandemic. Perspect Sex Reprod Health 52(2):75-79. https:// doi.org/10.1363/psrh.12151

28. Garrafa E, Levaggi R, Miniaci R, Paolillo C (2020) When fear backfires: emergency department accesses during the Covid-19 pandemic. Health Policy 124(12):1333-1339. https://doi.org/10. 1016/j.healthpol.2020.10.006

29. Griffith AM, Ockerse P, Shaaban A, Kelly C (2021) Effect of the COVID-19 pandemic on CT scans ordered from the emergency department for abdominal complaints. Emerg Radiol:1-11. https:// doi.org/10.1007/s10140-021-01907-4

Publisher's note Springer Nature remains neutral with regard to jurisdictional claims in published maps and institutional affiliations. 